\title{
Investigation into anti-epileptic effect and mechanisms of Ganoderma lucidum polysaccharides in in vivo and in vitro models
}

\author{
S. Zhou ${ }^{1,2}$, S.Q. Wang ${ }^{1}$, C. Y. Sun ${ }^{1}$, H.Y. Mao, W. H. Di ${ }^{1}$, X. R. Ma ${ }^{1}$, L. Liu ${ }^{1}$, J. X. Liu ${ }^{1}$, F. \\ F. Wang ${ }^{1}$, P. Kelly ${ }^{2}$ and P. Sreenivasaprasad ${ }^{2}$ \\ ${ }^{1}$ School of Basic Medical Sciences, Jiamusi University, Jiamusi 154000, P. R. China and ${ }^{2}$ Department of Life Science, \\ University of Bedfordshire, Luton, LU1 3JU, UK
}

There are about 60 million epileptic patients worldwide. Finding a more effective antiepileptic drug with fewer side effects is of continuing importance ${ }^{(1)}$. Ganoderma lucidum, a mushroom having been used as Traditional Chinese Medicine, has showed the effect of antiepileptic effect with no side-effects ${ }^{(2)}$. The aim of the present study was to assess the mechanisms of antiepileptic effects by Ganoderma lucidum polysaccharides (GLP).

In study 1 , effect of GLP on calcium turnover, $\mathrm{Ca}^{2+} /$ calmodulin-dependent protein kinase type II $\alpha$ (CaMKII $\left.\alpha\right)$ and ERK1/2 expression was investigated using primary hippocampal neurons from $<1$ day old rats. Neurons were cultured with normal medium (Control group), or $\mathrm{Mg}^{2+}$ free medium (Model I group) for 3 hours; neurons were incubated with $\mathrm{Mg}^{2+}$ free medium without GLP (Model II group), or with GLP $(0.375 \mathrm{mg} / \mathrm{ml})$ (GLP I group) for 3 hours and then cultured with the normal medium for an extra 3 hours; neurons were incubated with $\mathrm{Mg}^{2+}$ free medium for 3 hours and then cultured with a normal culture medium containing GLP for an extra 3 hours (GLP group II). In study 2, male Wistar rats $(n=60)$ were randomly divided into A) Control group; B) PBS group; and C) Epilepsy group: rats were given kainic acid $(1.5 \mu \mathrm{g} / \mu \mathrm{l}$ was injected into hippocampus), then they were given GLP (i.p.) once a day for 7 days, GLP doses were D), $25 \mathrm{mg} / \mathrm{kg}$; E), $50 \mathrm{mg} / \mathrm{kg}$ ), and F, $100 \mathrm{mg} / \mathrm{kg}$, respectively. Rat behavior and EEG were recorded. The number of immunohistochemical staining caveolin-1(Cav-1) and NF- $\mathrm{B}$ positive cells was counted.

In study 1, GLP treatment increased CaMKII $\alpha$ expression, and decreased calcium turnover. ERK1/2 had higher expression in cytoplasm and nucleus in Model groups compared with the normal Control groups $(P<0 \cdot 01)$. In GLP group, the expression of ERK1/2 (Fig. 1) was inhibited. In study 2. Epileptic symptoms in rats of group C, D, E and F were observed in 7 minutes after kainic acid injection. In day 7, normal EEG, with lower wavelength of $\alpha$ and $\beta$ wave, was recorded in group A and B; sharp and frequent wavelength was observed in group C, D, E or F (Fig. 2). Even more, compared to group C, more waves with lower wavelength existed in group D, E or F. The frequency of epilepsy discharge from initial 10-15/min reduced to $1 / \mathrm{min}$ after GLP treatment. Cav-1 positive

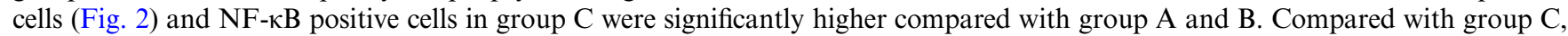
GLP treatments further increased Cav-1 positive cells, but decreased NF- $\mathrm{BB}$ positive cells.

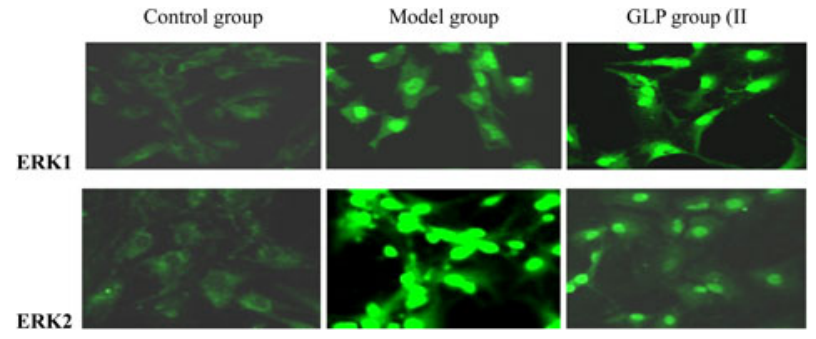

Fig. 1. Control group Model group GLP group (II)

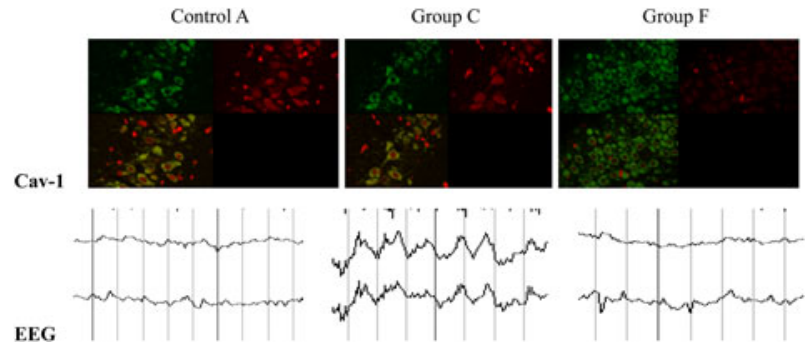

Fig. 2. Control A Group C Group F

In conclusion, GLP improved the epileptic behavior; inhibited calcium overloading and ERK1/2 and NF-KB expression; also stimulated CaMK II $\alpha$ and Cav-1 expression.

This work was supported by the National Natural Science Foundation, China (No. 81241112); Scientific and technological innovation on neurological cell injury and protection (2012TD013, Heilongiiang Province, China); Team focus on fostering technological innovation project funding (04099904, Jiamusi University, China)

1. Kobow K, Auvin S, Jensen F, et al. (2012) Epilepsia 53: 1868-1876.

2. Wang SQ, Li XJ, Zhou S, et al. (2013) PLoS One 8: E68. 